\title{
Preparation of Graphene-Copper Nanocomposite for Constructing Electrochemical Sensor for Paclitaxel Anti-Cancer Drug Detection in Taxus Chinensis
}

\author{
Yanjun Du ${ }^{1, *}$, Mengmeng Dou ${ }^{1}$, Wang $\mathrm{Ma}^{2}$, Xinjie Wang ${ }^{1}$, Zhaosen $\mathrm{u}^{1}$ and Xiaoming Deng ${ }^{1}$ \\ ${ }^{1}$ The First Affiliated Hospital of Zhengzhou University, Traditional Chinese Medicine Department, \\ No.1 Jianshe Eastern Road, Zhengzhou, Henan, China \\ ${ }^{2}$ The First Affiliated Hospital of Zhengzhou University, Oncology Department, No.1 Jianshe Eastern \\ Road, Zhengzhou, Henan, China \\ *E-mail: yanjundu_hn@qq.com
}

doi: $10.20964 / 2017.03 .77$

Received: 14 November 2016 / Accepted: 21 December 2016 / Published: 12 February 2017

In this work, a glassy carbon electrode modified with graphene and Au nanoparticles (GCE-grapheneEAu) was employed to electrochemically study paclitaxel which belonged to anticancer drug. Graphene and Au NPs were successfully electrodeposited on the surface of glassy carbon electrode in order to enhance the conductivity of electrode. An irreversible oxidation process was observed in the $\mathrm{pH}$ range of 3 to 10 , which was diffusion-controlled. A linear relationship was observed between the anodic peak current and the concentration of paclitaxel ranging from 0.01 to $2 \mathrm{mM}$, where the limits of detection (LOD) and quantification (LOQ) were 0.005 and $0.004 \mathrm{mM}$, respectively. The designed approach was demonstrated to be efficient in the determination of paclitaxel in Chinese herb Taxus chinensis.

Keywords: Taxus chinensis; Paclitaxel; Sensor; Au nanoparticles; Graphene

\section{$\underline{\text { FULL TEXT }}$}

(C) 2017 The Authors. Published by ESG (www.electrochemsci.org). This article is an open access article distributed under the terms and conditions of the Creative Commons Attribution license (http://creativecommons.org/licenses/by/4.0/). 